\title{
Check of the Performance of EDS Systems Attached to the SEM with the Test Material EDS-TM001/2 and Evaluation Software Package EDS Spectrometer Test - Application, Experiences and Updates
}

\author{
Vasile-Dan Hodoroaba ${ }^{1}$, Ralf Terborg ${ }^{2}$ and Mathias Procop ${ }^{1}$ \\ 1. Federal Institute for Materials Research and Testing (BAM), Division 6.1 Surface Analysis and \\ Interfacial Chemistry, 12200 Berlin, Germany. \\ 2. Bruker Nano GmbH, Am Studio 2D, 12489 Berlin, Germany.
}

The test material EDS-TM001 together with an accompanying software package, "EDX spectrometer check", have been made available in 2009 by BAM [1,2] to be employed by EDS (energy dispersive spectrometer) users to check the performance of an EDS attached to the SEM. Particularly for test laboratories operating under accreditation schemes like ISO/IEC 17025, a periodical control of the critical instrumental parameters in end-user laboratories is required. With EDS-TM001 or EDS-TM002 (second generation) test material, this periodical check is simplified to the acquisition of only one $10 \mathrm{kV}$ spectrum. The software "EDX spectrometer check" is destined to evaluate automatically this spectrum and determine the performance of the EDS in terms of energy resolution and calibration as well as possible alteration of low-energy efficiency due to detector contamination. Energy resolution can be compared with the specified values according to the international ISO standard ISO 15632:2012 [3]. EDS-TM is a synthetic material consisting of a thick layer of $\mathrm{C}, \mathrm{Al}, \mathrm{Mn}, \mathrm{Cu}$ and $\mathrm{Zr}$ in a well-defined composition, deposited on a steel (in case of EDS-TM001) or silicon (in case of EDS-TM002) substrate. Meanwhile, more than one hundred laboratories (most of them located in Germany) use the EDSTM001 or EDS-TM002 test material for the periodical check of their EDS. A detailed description of the test material and software together with examples of application was published recently [4]. New results and gained experiences will be presented as well.

When the FWHM of lines appearing in the EDS-TM spectrum are determined, the spectrum background must be subtracted accurately. The applied physical background subtraction procedure is robust and takes into account the transmission of the detector window [4]. While the previous version considers only Moxtek AP windows, the new version includes selection of silicon nitride window (with a transmission according to [5]) and the case of windowless detector, see Figure 1. Moreover, the new version allows importing of spectra in Bruker spx format and EMSA/MSA files from EDAX TEAM software.

Figure 2 shows the EDS-TM spectrum after background subtraction for a spectrometer with silicon nitride window and Figure 3 displays the corresponding results as provided by the updated "EDS Spectrometer Test" software. A spectrum corresponding to a windowless detector has been simulated with the DTSA-II software [6]. In this case, the appropriate background subtraction becomes particularly important, as the results in Table 1 show.

References:

[1] www.webshop.bam.de (Product: Reference materials, Category: Test materials).

[2] M Procop and V-D Hodoroaba, Microsc Microanal 15(Suppl 2) (2009), p. 1120. 
[3] ISO 15632:2012, "Microbeam Analysis - Selected instrumental performance parameters for the specification and checking of energy-dispersive X-ray spectrometers for use in electron probe microanalysis", ISO: Geneva.

[4] V-D Hodoroaba and M Procop, Microsc Microanal 20 (2014), p. 1556.

[5] http://amptek.com/products/c-series-low-energy-X-ray-windows/\#4.

[6] http://www.cstl.nist.gov/div837/837.02/epq/dtsa2/index.html

\begin{tabular}{|c|c|c|c|}
\hline Software version & FWHM C K /eV & FWHM Mn Ka /eV & Mn Lo/Mn Ko \\
\hline 3.4 and older & 54.7 & 128.6 & 1.43 \\
\hline 3.6 & 53.3 & 128.6 & 1.61 \\
\hline
\end{tabular}

Table 1. Results of the performance check for a windowless detector with older and new software versions based on a simulated spectrum (DTSA II).

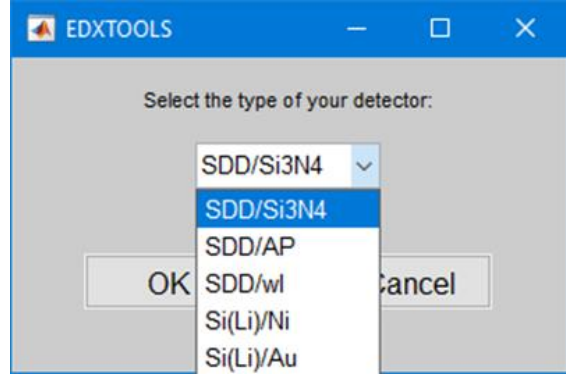

Figure 1. Pop-up menu for selection of the EDS detector type including the silicon nitride widow and windowless EDS.

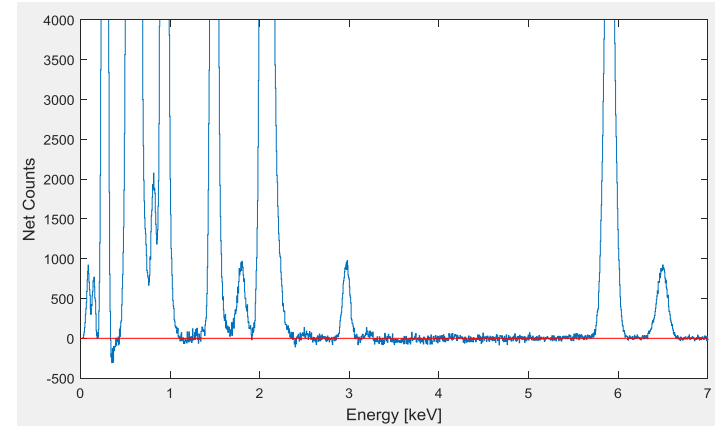

Figure 2. $10 \mathrm{kV}$ EDS-TM001 spectrum measured with a silicon nitride window SDD after background subtraction.

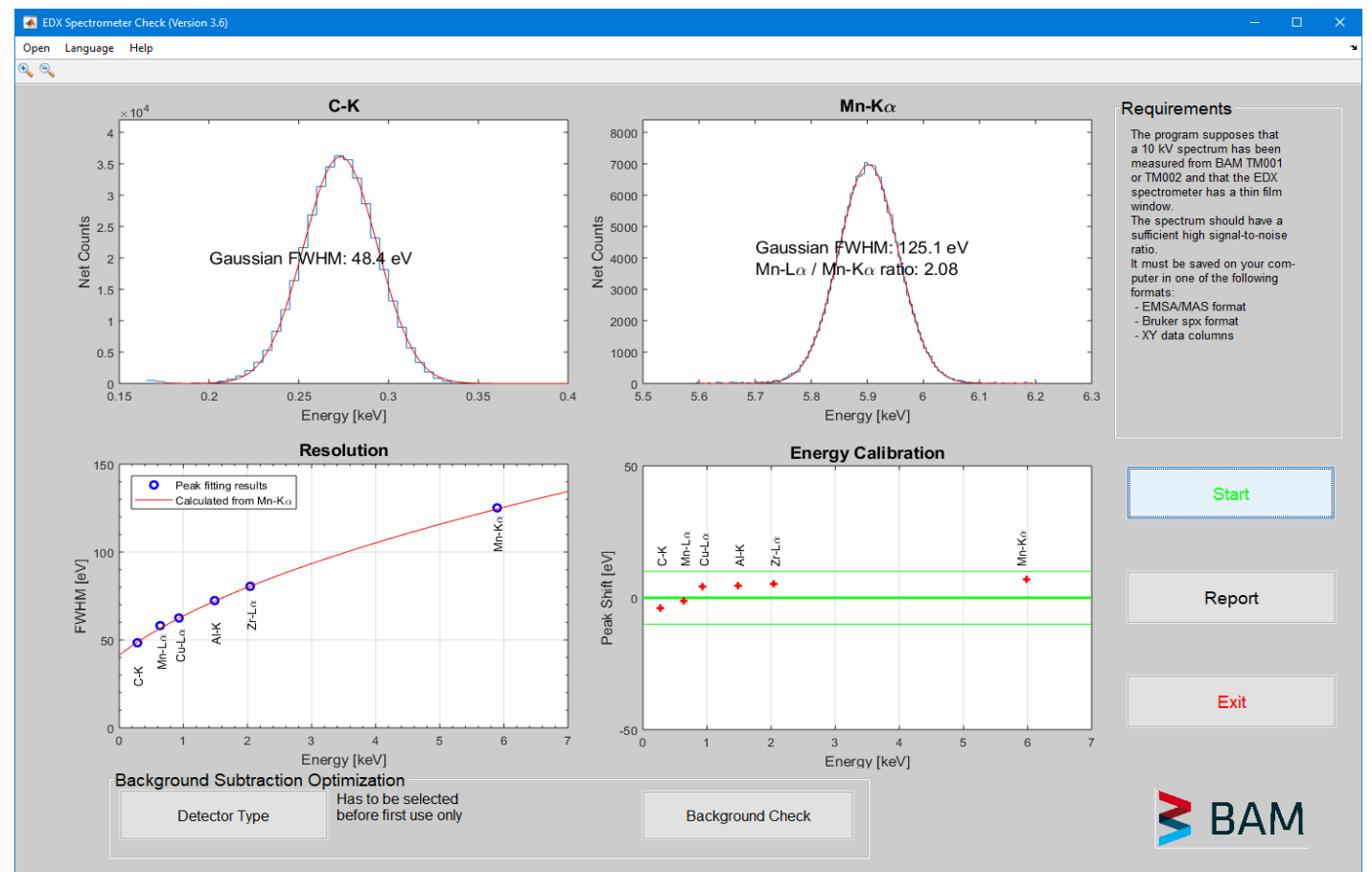

Figure 3. Result of evaluation of an SDD EDS with silicon nitride window with the updated software package "EDS Spectrometer Test", version 3.6 (release 2018). 